\title{
Parameter Estimation and Covariance Matrix of the Linear Functional Relationship Model for Circular Variables with Unequal Error Concentrations
}

\begin{abstract}
Nurkhairany Amyra Mokhtar ${ }^{1}$, Yong Zulina Zubairi ${ }^{2 *}$, Abdul Ghapor Hussin ${ }^{3}$ and Nor Hafizah Moslim4

${ }^{1,3}$ Faculty of Defence Sciences and Technology, National Defence University of Malaysia, Kem Sungai Besi, 57000 Kuala Lumpur, Malaysia.

${ }^{2}$ Centre for Foundation Studies in Science, University of Malaya, 50603 Kuala Lumpur, Malaysia. Institute of Graduate Studies, University of Malaya, 50603 Kuala Lumpur, Malaysia.

Functional relationship model is used to study the data that are subjected to errors. In this paper, we consider the linear functional relationship model with bivariate circular data where the pair of errors is with unequal concentration parameters. The parameter estimation of the model for circular data is different from linear data due to its wrapped around nature. We propose some improvements on the parameter estimation where some iterative procedures are considered. The concentration parameters are estimated based on the Bessel function. Also, we derive the corresponding covariance matrix of the model based on the Fisher Information matrix. Monte Carlo simulation studies were performed to study the suitability of the estimation method. It is found that the biasness of the estimates is small. Practical application of the method is illustrated by using real data set.
\end{abstract}

Keywords: circular data; covariance matrix; Von Mises distribution; simulation study

\section{INTRODUCTION}

A circular observation may be regarded as a point on a circle of unit radius. Each circular data is usually measured in degrees or in radians (Mardia \& Jupp, 2000). Due the wrapped around nature of angles, formal analysis cannot be done to circular data with usual statistical technique which is applied on linear data.

The early root of circular data analysis dates back at least as far as the mid-18th Century where discussion on the development of circular regression models began with Gould (1969), who predicted the mean direction of a circular response variable from a vector of linear covariates. Reverend John Mitchell FRS (1767) has analysed the angular separations between stars and made the hypothesis that the directions of the stars were uniformly distributed (Fisher, 1993). Other examples of circular data can be found in various scientific fields such as earth sciences, meteorology, biology, physics and medicine (Pandolfo et al., 2018).

The Von Mises distribution is said to be the most useful distribution for circular data (Mardia and Jupp, 2000) where Fisher (1987) noted that the Von Mises distribution is a symmetric unimodal distribution and characterised by a mean direction $\mu$ and concentration parameter $\kappa$. The probability density function of the distribution is:

$$
g(\theta ; \mu, \kappa)=\frac{1}{2 \pi I_{0}(\kappa)} e^{\kappa \cos (\theta-\mu)}
$$

where $I_{0}(\kappa)$ is the modified Bessel function of the first kind and order zero, which can be defined by: 


$$
I_{0}(\kappa)=\frac{1}{2 \pi} \int_{0}^{2 \pi} e^{\kappa \cos \theta} d \theta
$$

$$
\text { for } 0 \leq x<2 \pi, 0 \leq \pi<2 \pi \text { and } \kappa>0
$$

where $\mu$ is the mean direction and $\kappa$ is the concentration parameter.

In literature, circular variables may be studied using circular regression models when the error term is only considered for the $x$ variable; for example, in predicting direction of ground movement during an earth quake by Rivest (1997) and in medical imaging by Jones and Silverman (1989) (Down \& Mardia, 2002). As for the types of model, some examples of circular regression models are Jammalamadaka and Sarma model (1993), Down and Mardia model (2002), complex linear regression model for circular variables (Hussin et. al., 2010) and circular-circular regression model by Kato et al., (2008). In this paper, we consider the case when both $x$ and $y$ circular variables have error terms in linear functional relationship model.

Section II describes further about the linear functional relationship model for circular variables with unequal error concentration parameter, the maximum likelihood estimation of the parameters in the model, the derivation of the covariance matrix based on the Fisher information matrix and simulation study. Section III describes the results of the simulation studies, respectively. The applicability of the proposed method is described in Section IV. A concluding remark is given in Section V.

\section{MATERIALS AND METHODS}

\section{A. The Linear Functional Relationship Model for Circular Variables}

The concentration parameter $\mathcal{K}$ influences the Von Mises distribution $\operatorname{VM}(\mu, \kappa)$ inversely as $\sigma^{2}$ influences the normal distribution $N\left(\mu, \sigma^{2}\right)$ (Caires and Wyatt, 2003).

If in normal distribution, the variance $\sigma^{2}$ quantifies the dispersion (Fisher, 1993). However, in Von Mises distribution, the dispersion is quantified by a concentration parameter $\kappa$.

Functional relationship model is used to compare circular data subjected to errors. It is a type of error-in-variables model (EIVM) that considers error terms. There are some other models in EIVM which are structural relationship model and ultrastructural relationship model (Hassan et al., 2010). Equation (3) describes the Caires and Wyatt linear functional relationship model (LFRM) for circular variables.

$$
Y=\alpha+X(\bmod 2 \pi)
$$

In this model, both $X$ and $Y$ variables are subject to random errors $\delta_{i}$ and $\varepsilon_{i}$, respectively. Parameter estimation and the covariance matrix are derived for this model.

In this paper, the parameters of the LFRM, $X_{i}$, the rotation parameter $\alpha$ and the concentration parameter for $\delta$, which is $\kappa$, are estimated by using the method of maximum likelihood. Meanwhile the concentration parameter for $\varepsilon$, which is $v$, is estimated by using the ratio of $\lambda=\frac{v}{\kappa}$. Thus, $v=\lambda \kappa$. The log likelihood equation of the von Mises distribution for the LFRM is as given by:

$$
\begin{aligned}
& \log L(\alpha, \kappa, X ; x, y) \\
& =-2 n \log 2 \pi-n \log I_{0}(\kappa)-n \log I_{0}(\lambda \kappa) \\
& +\kappa \sum_{i=1}^{n} \cos \left(x_{i}-X_{i}\right)+\lambda \kappa \sum_{i=1}^{n} \cos \left(y_{i}-\alpha-X_{i}\right)
\end{aligned}
$$

\section{B. The Maximum Likelihood Estimation of} Parameters

The maximum likelihood estimation of the parameters involved in this model is discussed in the following subsections.

1. Maximum Likelihood Estimation of $X_{i}$

In this paper, we propose an improved estimate of $X_{i}$ where iterative procedure is considered on the incidental parameter. From the maximum likelihood estimation, we will be able to estimate the value of $X_{i}$ where $X_{i}=x_{i}+\partial_{i}$. From the log likelihood function in (4), we 
find its first derivative with respect to $X_{i}$ and set it equals to zero.

$\kappa \sum_{i=1}^{n} \sin \left(x-X_{i}\right)+\lambda \kappa \sum_{i=1}^{n} \sin \left(y_{i}-\alpha-X_{i}\right)=0$

We solve $X_{i}$ iteratively by some initial guess. Suppose $\hat{X}_{i 0}$ is an initial estimate of $\hat{X}_{i}$. Then

$$
x_{i}-\hat{X}_{i}=x_{i}-\hat{X}_{i 0}+\hat{X}_{i 0}-\hat{X}_{i}=\left(x_{i}-\hat{X}_{i 0}\right)+\Delta_{i}
$$

where $\Delta_{i}=\hat{X}_{i 0}-\hat{X}_{i}$.

We may also have

$$
y_{i}-\hat{\alpha}-\hat{X}_{i}=\left(y_{i}-\hat{\alpha}-\hat{X}_{i 0}\right)+\Delta_{i} \text {. }
$$

Hence, the partial derivative equation above becomes:

$$
\sin \left(x_{i}-\hat{X}_{i 0}+\Delta_{i}\right)+\sin \left(y_{i}-\hat{\alpha}-\hat{X}_{i 0}+\Delta_{i}\right)=0
$$

When $\Delta_{i}$ is small, then $\cos \Delta_{i} \approx 1$ and $\sin \Delta_{i} \approx \Delta_{i}$. It is worthwhile to note that the estimation is obtained iteratively where the ratio of the error concentration takes into account of the difference of the estimate at each iterative step. Hence the equation becomes:

$$
\hat{X}_{i 1} \approx \hat{X}_{i 0}+\frac{\sin \left(x_{i}-\hat{X}_{i 0}\right)+\lambda \sin \left(y_{i}-\hat{\alpha}-\hat{X}_{i 0}\right)}{\cos \left(x_{i}-\hat{X}_{i 0}\right)+\lambda \cos \left(y_{i}-\hat{\alpha}-\hat{X}_{i 0}\right)}
$$

where $\hat{X}_{i 1}$ is an improvement of $\hat{X}_{i 0}$.

\section{Maximum Likelihood Estimation of a}

To find the parameter estimate of the rotation parameter $\alpha$, we find the first derivatives of the log likelihood function with respect to $\alpha$ and and set it equals to zero to find $\hat{\alpha}$. Then we get:

$$
\hat{\alpha}=\left\{\begin{array}{l}
\tan ^{-1}\left\{\frac{S}{C}\right\} \quad \text { when } S>0, C>0 \\
\tan ^{-1}\left\{\frac{S}{C}\right\}+\pi \text { when } C<0 \\
\tan ^{-1}\left\{\frac{S}{C}\right\}+2 \pi \text { when } S<0, C>0
\end{array}\right.
$$

where $S=\sum_{i=1}^{n} \sin \left(y_{i}-\hat{X}_{i}\right)$ and $C=\sum_{i=1}^{n} \cos \left(y_{i}-\hat{X}_{i}\right)$

\section{Maximum Likelihood Estimation of $\kappa$ and $v$}

The log likelihood equation of the von Mises distribution for the LFRM is as given by equation (4). To find the estimate of the concentration parameter $\kappa$, we look for the first derivative of $\log$ likelihood function with respect to $\kappa$ and set it equals to zero. It is given by

$$
\begin{aligned}
& \frac{\partial \log L}{\partial \kappa}=-n \frac{I_{0}^{1}(\kappa)}{I_{0}(\kappa)}+\sum_{i=1}^{n} \cos \left(x_{i}-X_{i}\right) \\
& -n \frac{I_{0}^{1}(\lambda \kappa)}{I_{0}(\lambda \kappa)}+\lambda \sum_{i=1}^{n} \cos \left(y_{i}-\alpha-X_{i}\right)=0
\end{aligned}
$$

where $I_{0}(\kappa)$ is the modified Bessel function of the first kind and order zero and $\kappa$ is the concentration parameter.

Let $A(\kappa)=\frac{I_{0}^{1}(\kappa)}{I_{0}(\kappa)}=\frac{I_{1}(\kappa)}{I_{0}(\kappa)}$

$$
\begin{aligned}
& \text { Thus, }-n A(\kappa)+\sum_{i=1}^{n} \cos \left(x_{i}-X_{i}\right) \\
& -n A(\lambda \kappa)+\lambda \sum_{i=1}^{n} \cos \left(y_{i}-\alpha-X_{i}\right)=0 \\
& A(\kappa)+\lambda A(\lambda \kappa) \\
& =\frac{1}{n}\left\{\begin{array}{l}
\sum_{i=1}^{n} \cos \left(x_{i}-X_{i}\right) \\
+\lambda \sum_{i=1}^{n} \cos \left(y_{i}-\alpha-X_{i}\right)
\end{array}\right\}=c \\
& \text { where } A(\kappa)=1-\frac{1}{2 \kappa}-\frac{1}{8 \kappa^{2}}-\frac{1}{8 \kappa^{3}}+\ldots ;
\end{aligned}
$$

$I_{0}(\kappa)$ and $I_{1}(\kappa)$ are the asymptotic power series for the Bessel functions.

Simplifying the equation (12) using (13), it is then becomes:

$$
\begin{aligned}
A(\kappa)+\lambda A(\lambda \kappa)= & 1-\frac{1}{2 \kappa}-\frac{1}{8 \kappa^{2}}-\frac{1}{8 \kappa^{3}}+\lambda \\
& -\frac{1}{2 \kappa}-\frac{1}{8 \lambda \kappa^{2}}-\frac{1}{8 \lambda^{2} \kappa^{3}}=c
\end{aligned}
$$


Equation (24) is then simplified to become a cubic expression of

$$
8(1+\lambda-c) \kappa^{3}-8 \kappa^{2}-\left(1+\frac{1}{\lambda}\right) \kappa-\left(1+\frac{1}{\lambda^{2}}\right)=0
$$

However, the solution of $\kappa$ in (15) cannot be obtained directly. To overcome this, one can obtain the numerical approximation using readily built-in function such as polyroot function. It gives a real root and another two values are complex roots. The real root was chosen to be $\hat{\kappa}$. Caires and Wyatt (2003) noted that in circular case, the estimation of a concentration parameter needs to be corrected by dividing it by 2 . Therefore, the estimate of the concentration parameter of $\delta_{i}$ becomes $\tilde{\kappa}=\frac{\hat{\kappa}}{2}$. As for the concentration parameter for $\varepsilon_{i}$ which is $v$, is estimated by using the ratio of $\lambda=\frac{v}{\kappa}$. Thus, $\tilde{v}=\lambda \tilde{\kappa}$.

\section{The Derivation of the Covariance Matrix}

We derive the covariance matrix of this model by using the Fisher Information matrix. The expected values of the negative term of second derivatives of the log likelihood function of $L$ are obtained. The Fisher information matrix, $F$ for $\hat{X}_{1,} \hat{X}_{2}, \ldots, \hat{X}_{n}, \tilde{\kappa}$ and $\hat{\alpha}$ is given by:

$$
F=\left[\begin{array}{ccc}
R & 0 & W \\
0 & S & 0 \\
W^{T} & 0 & U
\end{array}\right]
$$

$R$ is an $n \times n$ matrix of $\mathrm{E}\left(-\frac{\partial^{2} \log L}{\partial X_{i}^{2}}\right) \quad$ and $\mathrm{E}\left(-\frac{\partial^{2} \log L}{\partial X_{i} \partial X_{j}}\right)$ which is:

$R=\left[\begin{array}{ccc}(1+\lambda) \tilde{\kappa} A(\tilde{\kappa}) & & 0 \\ 0 & \ddots & (1+\lambda) \tilde{\kappa} A(\tilde{\kappa})\end{array}\right]$,
$W$ is an $n \times 1$ matrix of $\mathrm{E}\left(-\frac{\partial^{2} \log L}{\partial X_{i} \partial \alpha}\right)$ which is $w=\left[\begin{array}{c}\lambda \tilde{\kappa} A(\tilde{\kappa}) \\ \vdots \\ \lambda \tilde{\kappa} A(\tilde{\kappa})\end{array}\right], U$ is $\quad$ a $\quad 1 \times 1$ matrix of $\mathrm{E}\left(-\frac{\partial^{2} \log L}{\partial \alpha^{2}}\right)$ which is $U=[\lambda \widetilde{\boldsymbol{\kappa}} \boldsymbol{n} A(\widetilde{\boldsymbol{\kappa}})]$, and $S$ is a $1 \times 1$ matrix of $\mathrm{jE}\left(-\frac{\partial^{2} \log L}{\partial \kappa^{2}}\right)$ which is $S=\left[n\left[(1+\lambda) A^{\prime}(\tilde{\kappa})\right]\right]$

The covariance matrix of $\hat{\alpha}$ and $\tilde{\kappa}$ is

$\operatorname{cov}\left[\begin{array}{c}\hat{\alpha} \\ \tilde{\kappa}\end{array}\right]=\left[\begin{array}{cc}S^{-1} & 0 \\ 0 & \left(U-W^{T} R^{-1} W\right)^{-1}\end{array}\right]$

where $\left(U-W^{T} R^{-1} W\right)^{-1}=(1+\lambda)\left(\frac{1}{\lambda \kappa n A^{\prime}(\tilde{\kappa})}\right)$

and $S^{-1}=\frac{1}{n\left[(1+\lambda) A^{\prime}(\tilde{\kappa})\right]}$.

Thus,

$\operatorname{cov}\left[\begin{array}{c}\tilde{\kappa} \\ \hat{\alpha}\end{array}\right]=\left[\begin{array}{cc}\frac{1}{n\left[(1+\lambda) A^{\prime}(\tilde{\kappa})\right]} & 0 \\ 0 & \frac{1+\lambda}{\lambda \kappa n A^{\prime}(\tilde{\kappa})}\end{array}\right]$

\section{Simulation Study}

A Monte Carlo simulation study is done for unreplicated LFRM and the ratio of concentration parameters of the errors is $\lambda=\frac{\kappa}{v}$. The parameters are estimated by using the proposed method. The number of simulations, $s$ is set to be 5000 for each simulation. Without loss of generality, the values of $X$ have been generated from the Von Mises distribution of $V M(2,3)$ and the true value of $\alpha=0.7854$. The values of the ratio of concentration parameters of the error considered in this study are $\lambda=0.6667,0.5,1.5,2$ and 5 where the pairs of values for the corresponding $\lambda$ are as stated as in Table 1.

Table 1. Values of $\kappa$ and $v$ for the each $\lambda$ 


\begin{tabular}{|l|l|l|}
\hline$\lambda$ & $\kappa$ & $V$ \\
\hline $\mathbf{0 . 6 6 6 7}$ & 12 & 8 \\
\hline $\mathbf{0 . 5}$ & 14 & 7 \\
\hline $\mathbf{1 . 5}$ & 10 & 15 \\
\hline $\mathbf{2}$ & 10 & 20 \\
\hline $\mathbf{5}$ & 10 & 50 \\
\hline
\end{tabular}

For each value of $\kappa$, the sample size $n=50,100$ and 150 are considered for the simulation. The bias measures used for the rotation parameter $\hat{\alpha}$ are mean, circular distance and mean resultant. The bias measures for theconcentration parameters are mean, estimate bias and estimated root mean square error. The followings are the measures.

\section{Biasness of $\hat{\alpha}$}

$>\quad$ Mean of circular parameter $\hat{\alpha}, \overline{\hat{\alpha}}$ :

$$
\overline{\hat{\alpha}}= \begin{cases}\tan ^{-1}\left(\frac{S}{C}\right) & \text { when } \mathrm{S}>0, \mathrm{C}>0 \\ \tan ^{-1}\left(\frac{S}{C}\right)+\pi & \text { when } \mathrm{C}<0 \\ \tan ^{-1}\left(\frac{S}{C}\right)+2 \pi & \text { when } \mathrm{S}<0, \mathrm{C}>0\end{cases}
$$

where $C=\sum_{j=1}^{s} \cos \left(\hat{\alpha}_{j}\right)$ and $S=\sum_{j=1}^{s} \sin \left(\hat{\alpha}_{j}\right)$
$>$ Circular distance, $d=\pi-|\pi-| \overline{\hat{\alpha}}-\alpha \mid$

$>$ Mean resultant length,

$R=\frac{1}{s} \sqrt{\left(\sum_{j=1}^{s} \cos \left(\hat{\alpha}_{j}\right)\right)^{2}+\left(\sum_{j=1}^{s} \sin \left(\hat{\alpha}_{j}\right)\right)^{2}}$

\section{Biasness of $\tilde{\kappa}$}

$>$ Mean of $\tilde{\kappa}, \overline{\tilde{\kappa}}=\frac{1}{s} \sum_{j=1}^{s} \tilde{\kappa}_{j}$

$>$ Estimated bias, $E B=\overline{\widetilde{\kappa}}-\kappa$

$>$ Estimated Root Mean Square Errors,

ERMSE $=\sqrt{\frac{1}{s} \sum_{j=1}^{s}\left(\tilde{\kappa}_{j}-\kappa\right)^{2}}$

\section{RESULTS AND DISCUSSION}

Table 2 to Table 4 show the results of the simulation study. From Table 2, as the value of $n$ increases, we can say that the circular mean of $\hat{\alpha}$ becomes closer to its true value. The value of circular distance of $\hat{\alpha}$ becomes closer to zero as $n$ increases meanwhile the mean resultant length of $\hat{\alpha}$ becomes closer to 1 when $n$ increases. These indicate that the estimation is good.

Table 2. Biasness of $\hat{\alpha}$ (True value $\left.=\frac{\pi}{4}=0.7854\right)$

\begin{tabular}{|c|c|c|c|c|}
\hline & $\boldsymbol{n}$ & Circular Mean & Circular Distance & Mean Resultant Length \\
\hline$\lambda=\mathbf{0 . 6 6 6 7}$ & 50 & 0.7843 & 0.0011 & 0.9978 \\
\hline$\kappa=\mathbf{1 2}$ & 100 & 0.7863 & 0.0009 & 0.9989 \\
\hline$\nu=\mathbf{8}$ & 150 & 0.7855 & 0.0001 & 0.9993 \\
\hline$\lambda=\mathbf{0 . 5}$ & 50 & 0.7857 & 0.0003 & 0.9977 \\
\hline$\kappa=\mathbf{1 4}$ & 100 & 0.7858 & 0.0004 & 0.9989 \\
\hline$\nu=7$ & 150 & 0.7850 & 0.0004 & 0.9993 \\
\hline$\lambda=\mathbf{1 . 5}$ & 50 & 0.7850 & 0.0004 & 0.9991 \\
\hline$K=\mathbf{1 0}$ & 100 & 0.7860 & 0.0006 & 0.9994 \\
\hline$\nu=\mathbf{1 5}$ & 150 & 0.7853 & 0.0001 & 0.9984 \\
\hline$\lambda=\mathbf{2}$ & 50 & 0.7863 & 0.0009 & 0.9992 \\
\hline$K=\mathbf{1 0}$ & 100 & 0.7848 & 0.0006 & 0.9995 \\
\hline$v=\mathbf{2 0}$ & 150 & 0.7855 & 0.0001 & \\
\hline
\end{tabular}


ASM Science Journal, Volume 13, 2020

\begin{tabular}{|l|l|l|l|l|}
\hline$\lambda=\mathbf{5}$ & 50 & 0.7845 & 0.0009 & 0.9987 \\
\hline$K=\mathbf{1 0}$ & 100 & 0.7857 & 0.0003 & 0.9994 \\
\hline $\boldsymbol{V}=\mathbf{5 0}$ & 150 & 0.7853 & 0.0001 & 0.9996 \\
\hline
\end{tabular}

Table 3. Biasness of $\tilde{\kappa}$

\begin{tabular}{|c|c|c|c|c|}
\hline & $n$ & Mean & Estimate bias & ERMSE \\
\hline$\lambda=0.6667$ & 50 & 12.3667 & 0.3667 & 2.6027 \\
\hline$\kappa=12$ & 100 & 11.9584 & -0.0416 & 1.6838 \\
\hline$v=8$ & 150 & 11.8761 & -0.1239 & 1.3812 \\
\hline$\lambda=0.5$ & 50 & 14.4337 & 0.4337 & 3.0057 \\
\hline$K=14$ & 100 & 13.9463 & -0.0537 & 2.0223 \\
\hline$v=7$ & 150 & 13.7981 & -0.2019 & 1.6077 \\
\hline$\lambda=\mathbf{1 . 5}$ & 50 & 10.3988 & 0.3988 & 2.2289 \\
\hline$\kappa=10$ & 100 & 10.0764 & 0.0764 & 1.4655 \\
\hline$V=15$ & 150 & 9.9715 & -0.0285 & 1.1913 \\
\hline$\lambda=\mathbf{2}$ & 50 & 10.4645 & 0.4645 & 2.2960 \\
\hline$\kappa=10$ & 100 & 10.0751 & 0.0751 & 1.4718 \\
\hline$V=\mathbf{2 0}$ & 150 & 9.9708 & -0.0292 & 1.1933 \\
\hline$\lambda=\mathbf{5}$ & 50 & 10.3931 & 0.3931 & 2.3280 \\
\hline$\kappa=10$ & 100 & 9.9946 & -0.0054 & 1.6767 \\
\hline$v=\mathbf{5 0}$ & 150 & 9.8825 & -0.1175 & 1.4587 \\
\hline
\end{tabular}

Table 3 and Table 4 show the biasness of $\tilde{\kappa}$ and $\tilde{v}$. Their means become closer to the true value as $n$ increases. The values of estimate bias are relatively small and the ERMSE decreases as $n$ decreases, thus suggesting that the parameter estimate is efficient.

Table 4: Biasness of $\tilde{v}$

\begin{tabular}{|c|c|c|c|c|}
\hline & $\boldsymbol{n}$ & Mean & Estimate bias & ERMSE \\
\hline$\lambda=\mathbf{0 . 6 6 6 7}$ & 50 & 8.2444 & 0.2444 & 1.7351 \\
\hline$\kappa=\mathbf{1 2}$ & 100 & 7.9723 & -0.0277 & 0.9208 \\
\hline$\nu=\mathbf{8}$ & 150 & 7.9174 & -0.0826 & 1.5028 \\
\hline$\lambda=\mathbf{0 . 5}$ & 50 & 7.2169 & 0.2169 & 1.0111 \\
\hline$K=\mathbf{1 4}$ & 100 & 6.9731 & -0.0269 & 0.8038 \\
\hline$\nu=7$ & 150 & 6.8990 & -0.1010 & 3.3433 \\
\hline$\lambda=\mathbf{1 . 5}$ & 50 & 15.5982 & 0.5982 & 2.1982 \\
\hline$K=\mathbf{1 0}$ & 100 & 15.1145 & 0.1145 & 1.7870 \\
\hline$\nu=\mathbf{1 5}$ & 150 & 14.9572 & -0.0428 & 4.5919 \\
\hline$\lambda=\mathbf{2}$ & 50 & 20.9290 & 0.9290 & 2.9436 \\
\hline$K=\mathbf{1 0}$ & 100 & 20.1503 & 0.1503 & 2.3866 \\
\hline$\nu=\mathbf{2 0}$ & 150 & 19.9416 & -0.0584 & \\
\hline
\end{tabular}




\begin{tabular}{|c|c|c|c|c|}
\hline$\lambda=\mathbf{5}$ & 50 & 51.9654 & 1.9654 & 11.6398 \\
\hline$\kappa=\mathbf{1 0}$ & 100 & 49.9729 & -0.0271 & 8.3837 \\
\hline$\nu=\mathbf{5 0}$ & 150 & 49.4124 & -0.5876 & 7.2933 \\
\hline
\end{tabular}

\section{APPLICATION TO REAL WIND DIRECTION DATA}

It is known that wind parameters have important influences on thermal rating. Thus, the knowledge of wind direction on a specific location contributes to accurate estimation of real power transmission capacity (Heckenbergerova et. al., 2015). The method above is illustrated by real wind direction data from Humberside Coast, UK, developed by UK Rutherford and Appleton Laboratories, with the sample size of 100. Variable $x$ is the data measured by the techniques of HF radar system. It uses pulse radar and operates at frequency of 24.2$27 \mathrm{MHz}$. Meanwhile the variable $y$ is measured by using the technique of anchored wave buoy.

Also, the Akaike Information Criterion (AIC) and Bayesian Akaike Information Criterion (BIC) are used to identify the optimum model in a class of competing models. The best approximating model is the one with minimum AIC and BIC in the class of competing models (Mutua, 1994). The formula of AIC is:

$A I C=-2 \ln ($ maximized likelihood for model) +2 (number of fitted parameter). Meanwhile the formula for BIC is:

$$
\begin{aligned}
& B I C=-2 \ln (\text { maximised likelihood for model) } \\
& +(\text { no. of fitted parameter) } \ln (\mathrm{n})
\end{aligned}
$$

In the application of the proposed method to the real data set, it is shown that the model with $\lambda=0.5$ and 2 has the smallest value of AIC and BIC. The estimation of the concentration parameters of $X$ and $Y$, which are $\tilde{\kappa}$ and $\tilde{v}$, respectively, may be reversible since Caires and Wyatt (2003) have argued that in connection with the desired symmetry of the functional relationship model, the quantity chosen to be $X$ or $Y$ are independent. This indicates that the best model for the data set is:

$Y=0.04844+X(\bmod 2 \pi)$.

Table 5. Mean and variance for parameter estimates of real data with AIC and BIC values

\begin{tabular}{|c|c|c|c|c|c|}
\hline$\lambda$ & $\hat{\alpha}$ (variance) & $\tilde{\kappa}$ (variance) & $\tilde{v}$ (variance) & AIC & BIC \\
\hline $\mathbf{0 . 5}$ & $0.04844(0.18593)$ & $26.9355(0.00818)$ & $13.4677(0.00205)$ & -112.204 & -104.388 \\
\hline $\mathbf{1 . 0}$ & $0.04826(0.32849)$ & $17.904(0.00515)$ & $17.904(0.00515)$ & -99.837 & -92.0215 \\
\hline $\mathbf{3} / \mathbf{2}$ & $0.04833(0.51442)$ & $14.9360(0.00316)$ & $22.4040(0.00711)$ & -104.132 & -96.3161 \\
\hline $\mathbf{2 . 0}$ & $0.04844(0.74373)$ & $13.4678(0.00205)$ & $26.9355(0.00818)$ & -112.204 & -104.388 \\
\hline
\end{tabular}

\section{CONCLUSION}

In this paper, we extend the idea when the variables $x$ and $y$ related by linear functional relationship model with unequal error concentrations. We derived the parameter estimates of the model where numerical approximation is used to find the cubic roots of the concentration parameters, from the Bessel function. Additionally, the covariance matrix of the parameters is derived based on Fisher information matrix and the estimation of $X$ was further improved using iterative procedure. The performance of the method is studied through a Monte Carlo simulation, and it is found that the biasness of the estimates is small, thus implying the feasibility of the method of estimation. The applicability of the method is illustrated by using a real data set in which to choose the best value for the ratio of concentration parameters, Akaike Information Criterion and Bayesian Information Criterion are considered. 


\section{ACKNOWLEDGEMENTS}

This study is supported by National Defence University of Malaysia and University of Malaya (Research Grant: GPFoo6H-2018).

\section{REFERENCES}

Caires, S. \& Wyatt, L. R. 2003, A Linear Functional Relationship Mutua, F. M. 1994, The use of the Akaike Information Model for Circular Data with an Application to the Assessment of Ocean Wave Measurement. American Statistical Assoc \& the Internal Biometric Society J. of Agricultural, Biological \& Environmental Statistics: 8 (2),153-169. Criterion in the identification of an optimum flood frequency model. Hydrological Sciences -Journal- des Sciences Hydrologiques: 39(3), 235-244.

Down, T. D. \& Mardia, K. V. 2002, Circular Regression, Biometrika: 89 (3), 683-697.

Pandolfo, G., Casale, G. \& Porzio, G. C. 2018, Testing Circular

Fisher N. I. 1993, Statistical Analysis of Circular Data, Antipodal Symmetry Through Data Depths. In Classification, (Big) Data Analysis and Statistical Cambridge Uni Press.

Fisher, N. I. 1987, Problems with the Current Definitions of the Standard Deviation of Wind Direction. Journal of Climate and Applied Meteorology: 26, 1522-1529.

Gould, A. L. 1969, A Regression Technique for Angular Variates. Biometrics: 25(4), 683-700.

Hassan, S. F, Hussin, A. G. \& Zubairi, Y. Z. 2010, Estimation of Functional Relationship Model for Circular Variables \& Its Application in Measurement Problem, Chiang Mai J. Sci: 37(2), 195-205.

Heckenbergerova, J., Musilek, P., \& Krömer, P. 2015, Optimization of wind direction distribution parameters using particle swarm optimization. In Afro-European Conference for Industrial Advancement (pp. 15-26). Springer, Cham.

Hussin, A.G, Hassan, S. F. \& Zubairi, Y.Z. 2010, A Statistical Method to Describe the Relationship of Circular Variables Simultaneously. Pakistan Journal of Statistics: 26 (4), 593607.

Jammalamadaka, S. R. \& Sarma, Y. R. 1993, Circular regression. In: Matsusita, K., ed. Statistical Science and Data Analysis. Utrecht: VSP, pp. 109-128.

Kato S., Shimizu K. \& Shieh G. S. 2008, A Circular-circular Regression Model, StatisticaSinica: 18,633-645.

Mardia K. V. \& Jupp P. E., 200o, Directional Statistics, John Wiley \& Sons. 